\title{
Stimulus-Driven and Goal-Driven Control Over Visual Selection
}

\author{
Casimir J. H. Ludwig and Iain D. Gilchrist \\ University of Bristol
}

\begin{abstract}
This article explored the extent to which stimulus-driven control over visual selection is modulated by goal-driven factors. Observers searched for a no-onset color target among 3 distractors and signaled its location either manually or with a saccade. Additional distractors appeared either with or without an abrupt onset and were either similar or dissimilar to the target. Abrupt onsets disrupted saccades to the target, especially when they shared the target color. Irrelevant onsets also interfered with the manual responses, but this interference was dependent on the particular type of manual response. Stimulus-driven and contingent capture can occur within a single paradigm, but the extent and nature of these effects depend on the specific response required.
\end{abstract}

Theories of visual attention generally distinguish stimulusdriven selection from goal-driven selection (Egeth \& Yantis, 1997). The former indicates that attention is captured by a visually salient object, irrespective of the observer's intentions. The latter indicates the deliberate allocation of attention to objects that are goal-relevant in a given behavioral context.

Stimulus-driven attentional capture can be studied by having one of the items in a visual search display stand out on some irrelevant dimension (Simons, 2000). This salient item is no more (or less) likely to be the target than any other display item, so there is no incentive to specifically attend to it. If search is speeded up when the salient item happens to be the target, or slowed down when it is one of the distractors, it is thought to have captured attention. Using this rationale, various studies have demonstrated that items that appear in the visual scene with an abrupt onset capture attention automatically (Theeuwes, 1994; Yantis \& Jonides, 1984, 1990). Several reasons have been put forward as to why this may be so, including changes in luminance or other primary perceptual dimensions (Gellatly, Cole, \& Blurton, 1999; Yantis \& Jonides, 1984), forward masking of the no-onset elements (Gibson, 1996a, 1996b), and appearance of a new object (Gellatly \& Cole, 2000; Yantis \& Hillstrom, 1994).

However, stimulus-driven capture by abrupt onsets is not inevitable, and substantial effort has been directed toward determining the extent to which capture is subject to top-down control. For instance, Yantis and Jonides (1990) have shown that abrupt onsets fail to attract attention automatically when a (valid) precue indicates the target location. Bacon and Egeth (1994) argued that for capture by irrelevant singleton distractors to occur, the attentional

Casimir J. H. Ludwig and Iain D. Gilchrist, Department of Experimental Psychology, University of Bristol, Bristol, Great Britain.

This work was supported by a University of Bristol postgraduate research scholarship awarded to Casimir J. H. Ludwig. We thank three anonymous reviewers for their helpful comments and suggestions on earlier versions of this article.

Correspondence concerning this article should be addressed to Casimir J. H. Ludwig, Department of Experimental Psychology, University of Bristol, 8 Woodland Road, Bristol BS8 1TN, Great Britain. E-mail: c.ludwig@bristol.ac.uk system had to be in a singleton detection mode (which, in many of these search experiments, would be an efficient strategy for target selection). Top-down control overrides stimulus-driven capture when such a strategy is rendered useless and observers are set to search for a value on some specific target dimension or dimensions.

The idea that capture is dependent on attentional control settings is at the heart of the contingent capture hypothesis put forward by Folk, Remington, and colleagues (Folk, Remington, \& Johnston, 1992; Folk, Remington, \& Wright, 1994). In their cuing paradigm, the cost (in terms of manual reaction time) of invalidly indicating the target location was dependent on whether the cue shared properties with the target. For instance, when the target was defined by an abrupt onset, there was a cost of presenting invalid abrupt-onset cues but not of color cues. They claimed that capture is never purely stimulus-driven and occurs only when the salient, irrelevant item has properties to which the system is set up to respond.

The debate on attentional control over capture is ongoing and far from settled. One problem is that the different paradigms that have been adopted to investigate these issues have led to different conclusions (Remington, Folk, \& McLean, 2001; Simons, 2000). Thus, pure stimulus-driven capture has been found in search tasks, whereas contingent capture has mainly been shown in the attentional cuing paradigm. One solution to this problem is to use a visual search task that allows for the assessment of the extent to which stimulus-driven capture is contingent on top-down control settings. With this aim, in the current experiments we tested the control over capture in a type of visual search paradigm that has been shown to be effective in eliciting stimulus-driven capture.

Research into stimulus-driven attentional capture has recently been extended to the oculomotor domain by Theeuwes and colleagues (Theeuwes, Kramer, Hahn, \& Irwin, 1998; Theeuwes, Kramer, Hahn, Irwin, \& Zelinsky, 1999). Search for a no-onset color target was slowed down when an additional distractor appeared with an abrupt onset. In addition, observers frequently made an inappropriate saccade to the onset before redirecting gaze to the target.

As yet, there has been little investigation of contingent capture in the oculomotor domain. Irwin, Colcombe, Kramer, and Hahn (2000) noted that although observers were set for a specific color 
target they were still captured by abrupt-onset distractors. Irwin et al. argued that this finding was inconsistent with the contingent capture hypothesis. However, because the target had a unique color in their study, as in Theeuwes et al. (1998, 1999), one could maintain that observers were set in a singleton detection mode. These results, therefore, do not necessarily argue against contingent capture.

By investigating top-down control over capture not only for manual but also for saccadic responses, the current experiments extend the contingent capture research into the oculomotor domain. Comparing manual and saccadic responses to the same search stimuli directly addresses the issue of whether visual selection is subserved by a common spatial representation of salience that can be drawn on regardless of the response (an assumption that is implicit in much research on visual attention and in computational models of visual selection) or whether the putative salience map is influenced by motor output factors (Briand, Larrison, \& Sereno, 2000).

In summary, in the current series of experiments we investigated two questions. First, are contingent capture and stimulus-driven capture restricted to specific paradigms, or can both effects be observed in a single experiment? Second, what is the relative contribution of stimulus-driven and goal-driven factors for different responses (manual and saccadic)?

We addressed the first question by manipulating the target similarity of additional onset distractors. Observers responded to a color target while on some trials an additional distractor was presented in one of two locations known to be irrelevant. This distractor appeared either with or without an abrupt onset and shared its color with either the target or the distractors. Note that because similar distractors appeared on a fair amount of trials, the target could not be localized reliably by responding to (color) singletons, which should have discouraged participants from entering a singleton detection mode (cf. Bacon \& Egeth, 1994). According to the contingent capture hypothesis, capture should occur only when the additional distractor is similar to the target, irrespective of whether it has an abrupt onset (because this is not a property that the system is set to respond to). In separate blocks of trials, the impact of the additional distractor was determined for manual and saccadic responses. Thus, conclusions derived from different responses to exactly the same stimulus input could be directly compared (within participants). In Experiment 1, a button press indicating the target quadrant was compared with locating the target with a saccade.

\section{Experiment 1}

\section{Method}

Participants. Twelve observers ( 2 men and 10 women; age range: $18-34$ years) took part in the experiment for course credits or payment. All had self-reported normal or corrected-to-normal vision and were able to discriminate the colors used in this experiment.

Materials and stimuli. Placeholder displays consisted of a central, gray circular disk (with a radius of $0.3^{\circ}$ ) that served as the fixation point and four placeholders indicating the possible target locations (on trials with an additional no-onset distractor, a fifth placeholder indicated the location of the upcoming extra distractor). Placeholders were gray vertical bars subtending $0.6^{\circ} \times 1.9^{\circ}$. Placeholders and fixation point were of the same chromaticity (CIE $x, y$ chromaticity coordinates of .28/.30) and luminance $\left(7.6 \mathrm{~cd} / \mathrm{m}^{2}\right)$ and were presented on a black background $\left(0.0 \mathrm{~cd} / \mathrm{m}^{2}\right)$. The four placeholders that indicated the possible target locations formed an imaginary rectangle centered on fixation. The additional distractor (onset or no onset) could appear left or right of fixation, on the horizontal midline. Thus, there were six positions in which elements could be presented; these six locations were arranged on the circumference of an imaginary circle around fixation with a radius of $7.3^{\circ}$. The search displays consisted of red (CIE coordinates of .63/.33) and green (CIE coordinates of .29/.59) vertical bars of the same size and similar luminance as the placeholders.

Displays were presented on a $17-$ in. $(43.18-\mathrm{cm})$ SVGA monitor with $800-\times 600$-pixel resolution and $74-\mathrm{Hz}$ refresh rate. The monitor was located $57 \mathrm{~cm}$ from the chin rest. A second PC was used to record eye position data on-line. Eye movements were monitored with the SMI EyeLink System (SensoMotoric Instruments GmbH, Teltow, Germany). The system uses the center of the pupil and the corneal reflection to define pupil position. Eye movements were recorded at $250 \mathrm{~Hz}$, with an operational spatial resolution of about $0.3^{\circ}$. Saccade onset was defined as a change in eye position with a minimum velocity of $35 \%$ or a minimum acceleration of $9500^{\circ} / \mathrm{s}$.

Procedure. Each of the two blocks of trials started with a nine-point grid calibration and validation procedure. Participants were asked to saccade to a gray, circular disk (identical to the fixation point) that appeared sequentially (but unpredictably) in a $3 \times 3$ grid. After a satisfactory validation had been obtained, a block of trials was run.

Participants searched for the red target among green distractors. A different response to the target was required for each block. In the manual response block, participants placed their thumbs and forefingers on four buttons in a rectangular arrangement and signaled the location of the target by pressing the corresponding button as quickly as possible. Participants were instructed to maintain fixation throughout this block. In the saccadic response block, participants signaled the target location by foveating the target as quickly as possible. The order of these two blocks was counterbalanced across participants.

On two thirds of the trials in a block, an additional distractor appeared, either left or right of fixation on the horizontal midline. This additional distractor was completely irrelevant to the task, and participants were instructed to ignore it. It could be either similar (in fact, identical) or dissimilar (but similar to the other distractors) to the target. In addition, it could appear either in a location previously occupied by an extra placeholder (no-onset distractor) or in a previously empty location (onset distractor). Combining the similarity and onset/no-onset factors created four trial types: similar onset, similar no onset, dissimilar onset, and dissimilar no onset. These four trial types were equally distributed over the trials with an additional distractor (thus, one sixth of all trials within a block). The remaining trials in a block (one third) were baseline trials on which no additional distractor was presented. Each of the four baseline display configurations (the target appeared in four positions) was combined equally often with left and right, similar and dissimilar onset and no-onset distractors. A block consisted of 144 trials. The different trial types were randomly intermixed in a block.

In between trials a fixation display was presented, consisting of just the central fixation disk. When the observer had properly fixated, the experimenter initiated a new trial, and, if necessary, an automatic spatial drift correction was performed. An experimental trial began with the 600-ms presentation of the placeholders, followed by the search display. The search display remained visible for $1 \mathrm{~s}$, during which participants were required to respond as quickly and accurately as possible. Typical examples of placeholder and search displays for the different trial types are shown in Figure 1.

Data analysis. Trials on which the central disk was not properly fixated (deviation $>2^{\circ}$ ) at presentation of the search display were excluded from analysis. For the manual response block, trials on which observers made a saccade before the button press were rejected. Moreover, anticipatory $(<100 \mathrm{~ms})$ and delayed $(>1,000 \mathrm{~ms})$ manual responses were not 


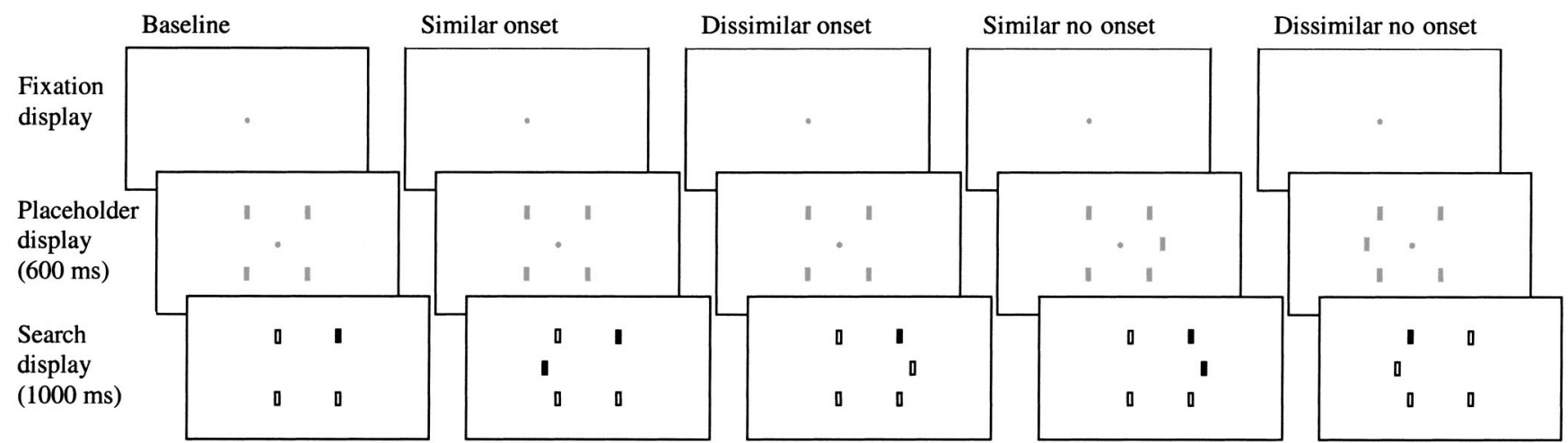

Figure 1. The basic sequence and time course of the displays in Experiment 1. Placeholders are indicated by the gray bars. Bars that appeared in a distractor color are shown in white, and bars that appeared in the target color are shown in black.

analyzed. In the saccadic response block, the shortest latency with which observers directly fixated the target with a probability above chance was $125 \mathrm{~ms}$. Therefore, trials on which observers made a saccade with a latency shorter than $125 \mathrm{~ms}$ were considered anticipatory and were excluded from further analysis (this criterion for anticipatory saccades was then used in all subsequent experiments). Moreover, trials with no saccade, too small a saccade $\left(<2^{\circ}\right)$, or too large a saccade $\left(>12^{\circ}\right)$ were rejected.

Mean correct manual reaction times (MRTs) were entered in repeated measures analyses of variance (ANOVAs) with onset and similarity (similar or dissimilar to the target) of the additional distractor as two-level, within-participant factors. (Unless stated otherwise, these factors were entered in each ANOVA reported throughout this article.)

To determine the landing position of the first saccade, the imaginary circle on which the display elements were positioned was divided in eight $45^{\circ}$ segments, six of which could contain a display element. If the amplitude of the saccade was between $2^{\circ}$ and $12^{\circ}$, the saccade was classified as having landed on the target, on the additional distractor, on any of the other distractors, or in an empty display segment. Because the vast majority of saccades were directed either to the target or to the additional distractor, the few saccades that landed in an empty segment or on one of the other distractor items were excluded from further analysis. That is, the proportions of first saccades landing on the target were rescaled, assuming that first saccades went either to the target or to the additional distractor. These proportions were then transformed into corrected arcsine values (Anscombe, 1948). Such a transformation was appropriate, because in some conditions the proportions were close to the ends of the percentage scale. The transformation served to improve the normality and homogeneity of variance of the scores. The transformed values were then entered into repeated measures ANOVAs similar to the MRTs. Nonparametric post hoc testing of the percentage of target-directed saccades was performed using the Wilcoxon signed-ranks test. The mean correct saccadic latencies were dealt with in the same way as the MRTs.

\section{Results}

Manual responses. For each participant, we excluded the first trial from the analysis. In addition, $0.8 \%$ of the trials were rejected because of improper fixation, a further $1.8 \%$ because of misses, $0.7 \%$ as a result of incorrect manual responses, and $12.7 \%$ as a result of eye movements larger than $2^{\circ}$.

The mean correct MRTs for the different trial types are shown in Table 1. Only the effect of similarity was significant, $F(1,11)=$ $84.33, p<.01$. Responses were slower if the additional distractor was similar to the target, whether it appeared with an abrupt onset or no onset (an increase of 70 and $77 \mathrm{~ms}$, respectively). The comparison that came closest to inspection of display size effects in this search task was that between the baseline and dissimilar no-onset trials. Adding a dissimilar distractor without an abrupt onset was not associated with an MRT cost (increase of $1 \mathrm{~ms}$ ), $t(11)=0.29, p>.05$. Thus, target selection was highly efficient

Table 1

Manual and Saccadic Response Data (in Milliseconds) From Experiment 1

\begin{tabular}{lccccc}
\hline & \multicolumn{5}{c}{ Trial type } \\
\cline { 2 - 6 } $\begin{array}{c}\text { Response type } \\
\text { and measure }\end{array}$ & Baseline & $\begin{array}{c}\text { Similar } \\
\text { onset }\end{array}$ & $\begin{array}{c}\text { Similar } \\
\text { no onset }\end{array}$ & $\begin{array}{c}\text { Dissimilar } \\
\text { onset }\end{array}$ & $\begin{array}{c}\text { Dissimilar } \\
\text { no onset }\end{array}$ \\
\hline $\begin{array}{l}\text { Manual } \\
\quad \text { Mean correct MRT }\end{array}$ & 443 & 527 & 521 & 457 & 444 \\
$\begin{array}{l}\text { Saccadic } \\
\text { Errors }\end{array}$ & - & 0.49 & 0.19 & 0.07 & \\
$\quad$ SRT correct & 244 & 299 & 281 & 246 & 245 \\
$\quad$ SRT errors & - & 222 & 251 & 213 & \\
\hline
\end{tabular}

Note. Errors are the proportions of responses directed to the additional distractor. Dashes indicate no errors could be made in the trials (only applicable to baseline trials). MRT = manual reaction time; SRT $=$ saccadic reaction time. 
on these trials, as would be expected in a standard feature pop-out search (Treisman \& Gelade, 1980).

Participants found it difficult to maintain fixation throughout the trials, as evidenced by the $12.7 \%$ of trials rejected because of eye movements larger than $2^{\circ}$. Given that eye movement programming takes time, it was possible that the MRTs on these trials were prolonged relative to those trials on which participants succeeded in maintaining fixation. Thus, excluding these trials might have affected the pattern of results obtained. However, note that the percentage of rejections did not systematically vary over the different trial types $(12.3 \%$ of the baseline trials, $12.9 \%$ of the similar onset trials, $13.2 \%$ of the similar no-onset trials, $17.0 \%$ of the dissimilar onset trials, and $10.1 \%$ of the dissimilar no-onset trials). Indeed, when these trials were included in the analysis, again only the effect of similarity was significant, $F(1,11)=70.26, p<.01$.

Saccadic responses. As in the manual response condition, we excluded the first trial from further analyses. Of the remaining trials, $2.9 \%$ were rejected because of improper fixation at presentation of the search display, $1.3 \%$ because the eye movement was anticipatory, and $7.0 \%$ because the amplitude of the saccade was either too small or too large. Almost all first saccades landed on either the target or the additional distractor; only $0.9 \%$ landed on one of the other distractors or in an empty segment.

Table 1 lists the proportion of first saccades to the additional distractor. It is clear from these error proportions that the degree to which abrupt onsets elicited an inappropriate eye movement was mediated by target similarity. The two main effects of similarity and onset were significant, $F(1,11)=75.38, p<.01$, and $F(1,11)$ $=29.57, p<.01$, respectively. In addition, the interaction between similarity and onset was significant, $F(1,11)=8.53, p<.05$. Post hoc (Wilcoxon signed-ranks) tests revealed that similar onset distractors captured the eyes more often than similar no onsets, $T(12)=0.00, p<.01$, and dissimilar onset distractors, $T(12)=$ $0.00, p<.01$. Dissimilar onsets elicited more saccades than their no-onset counterparts, $T(7)=0.00, p<.05$. The interaction between similarity and onset stemmed from a larger effect of onset when the distractor was similar to the target. However, it is not clear how to interpret the interaction, given the small number of first saccades to the dissimilar no-onset distractor.

In the analysis of the mean correct saccadic latencies, only the effect of similarity was significant, $F(1,11)=27.16, p<.01$. Latencies were increased in the presence of a similar additional distractor, irrespective of whether it had an abrupt onset. The latencies of eye movements to the additional distractor were generally shorter than those of the target-directed saccades. The shortest reaction times were observed for saccades to irrelevant onsets.

\section{Discussion}

The MRT data showed no indication of attentional capture by onset distractors when participants were searching for a target defined by color. There was interference only when the additional distractor was similar to the target, irrespective of whether it had an abrupt onset. This finding fits well with the contingent capture hypothesis of Folk et al. (1992, 1994). In their experiments, when observers were set to search for a target defined by color, there was only a cost of presenting invalid color cues but not of invalid abrupt-onset cues. In contrast, the accuracy of the eye movements did reveal stimulus-driven capture by abrupt onsets, over and above the interference caused by similar additional distractors.

In Experiment 1, a direct comparison between onset and noonset trials may not have been completely valid: On no-onset trials, the extra placeholder reliably cued the presence and location of an additional distractor. Thus, in comparison with onset trials, observers had to inhibit, or visually mark (Watson \& Humphreys, 1997), only one position instead of two (and it may be more difficult to inhibit empty locations than locations already occupied by an object). To test for this possible interpretation of the differences between the onset and no-onset conditions and to replicate the basic effects, in Experiment 2 there were always five placeholders, and the extra placeholder did not predict whether and where an extra, irrelevant item would appear. In all other respects, Experiment 2 was identical to Experiment 1.

\section{Experiment 2}

\section{Method}

Participants. Twelve new observers ( 3 men and 9 women; age range: 18-34 years) were recruited. All had self-reported normal or corrected-tonormal vision and were able to discriminate the colors used in this experiment.

Materials and stimuli. The same equipment was used as in Experiment 1. As for the stimuli, the only difference was that the placeholder display always contained five gray vertical bars: the four that formed an imaginary rectangle and indicated the possible target location, and a fifth one in one of the two distractor locations. On baseline trials the additional placeholder simply disappeared, on onset trials it disappeared and either a red (similar to the target) or a green (dissimilar) element appeared on the opposite side, and on no-onset trials it changed from gray to either red or green (similar or dissimilar no onset). A block contained 154 trials, the first 10 of which were considered practice. The distribution of the remaining 144 trials over the different trial types was identical to that in Experiment 1.

Procedure. In two separate blocks of trials, observers signaled the location of the red target among green distractors either with a speeded button press or with a saccade.

\section{Results}

Manual responses. Improper fixation resulted in the rejection of $0.8 \%$ of the trials. In addition, $7.8 \%$ of the trials were rejected because of delayed responses (misses) and $13.4 \%$ because a saccade was made. Incorrect responses occurred only rarely $(0.8 \%)$; these trials were also excluded from further analyses.

The mean correct MRTs are shown in Table 2. A similar pattern as that in Experiment 1 was obtained, with only the main effect of similarity reaching significance, $F(1,11)=41.76, p<.01$. Again, responses to the target were slowed down in the presence of an additional distractor that was similar to the target, irrespective of whether it was presented with an abrupt onset (57-ms and 59-ms cost, respectively). The 4-ms increase in MRT between baseline and dissimilar no-onset trials was nonsignificant, $t(11)=0.47, p>$ .05 , confirming again that target selection under these conditions was highly efficient.

As in Experiment 1, participants did not always maintain fixation- $13.4 \%$ of the trials were rejected on this basis, including $19.8 \%$ of the baseline trials, $16.7 \%$ of the similar onset trials, $12.5 \%$ of the similar no-onset trials, $11.5 \%$ of the dissimilar onset 
Table 2

Manual and Saccadic Response Data (in Milliseconds) from Experiment 2

\begin{tabular}{lccccc}
\hline & \multicolumn{5}{c}{ Trial type } \\
\cline { 2 - 6 } $\begin{array}{c}\text { Response type } \\
\text { and measure }\end{array}$ & Baseline & $\begin{array}{c}\text { Similar } \\
\text { onset }\end{array}$ & $\begin{array}{c}\text { Similar } \\
\text { no onset }\end{array}$ & $\begin{array}{c}\text { Dissimilar } \\
\text { onset }\end{array}$ & $\begin{array}{c}\text { Dissimilar } \\
\text { no onset }\end{array}$ \\
\hline $\begin{array}{l}\text { Manual } \\
\quad \text { Mean correct MRT }\end{array}$ & 464 & 532 & 527 & 475 & 468 \\
$\begin{array}{l}\text { Saccadic } \\
\text { Errors }\end{array}$ & - & 0.47 & 0.28 & 0.05 & \\
$\quad$ SRT correct & 228 & 269 & 268 & 234 & 234 \\
$\quad$ SRT errors & - & 206 & 229 & 171 & \\
\hline
\end{tabular}

Note. Errors are the proportions of responses directed to the additional distractor. Dashes indicate no errors could be made in the trials (only applicable to baseline trials). MRT = manual reaction time; $\mathrm{SRT}=$ saccadic reaction time.

trials, and none of the dissimilar no-onset trials. The analysis of mean correct MRTs with these trials included also showed only an effect of similarity, $F(1,11)=42.67, p<.01$.

Saccadic responses. Of all trials in this condition, $4.2 \%$ were excluded because of improper fixation, $4.3 \%$ because the saccade was either too small or too large, and $1.6 \%$ as a result of anticipatory saccades. The majority of first saccades landed either on the target or on the additional distractor. Only $0.9 \%$ landed on one of the other distractors or in an empty segment.

As can be seen in Table 2, the distribution of distractor-directed saccades over the different trial types was similar to that of Experiment 1 . There were main effects of similarity and onset, $F(1,11)=83.39, p<.01$, and $F(1,11)=8.37, p<.05$, respectively. However, in contrast to the previous experiment, the interaction between the two factors was not significant, $F(1,11)=$ $1.81, p>.05$, probably because the effect of onset was smaller for the similar additional distractors. Post hoc comparisons showed that similar onsets captured the eyes more frequently than similar no-onset distractors, $T(12)=9.50, p<.05$, and dissimilar onsets, $T(12)=1.00, p<.01$. The difference between dissimilar onset and no-onset distractors did not reach significance, $T(3)=0.00$, $p=.11$.

Analysis of the mean correct latencies revealed that targetdirected saccades had longer latencies when initiated in the presence of a similar distractor, irrespective of whether it had an abrupt onset, $F(1,11)=79.23, p<.01$. The incorrect latencies were generally shorter, particularly when the saccade was directed to an abrupt-onset distractor.

Combined analysis: Experiments 1 and 2. The patterns of results in the first two experiments were very similar. One of the strong conclusions across the two experiments is that the manual responses showed interference only from similar additional distractors: There was no reliable effect of onset in either experiment. However, inspection of Tables 1 and 2 shows that the manual responses were generally somewhat slower in trials with an onset distractor. This finding suggests that there may be a small effect of onset that neither experiment alone had the statistical power to detect. To formally investigate this effect, we carried out a mixedfactor ANOVA on the mean correct MRTs, with similarity and onset as the two within-participant factors and experiment as the one between-participant factor. This analysis also constituted a direct test of the effect of the extra placeholder. In Experiment 2, the extra placeholder was made unpredictive of the presence and location of the additional distractor. As a result, on baseline trials and (more importantly) onset trials, the extra placeholder disappeared. Thus, in comparison with the first experiment, these trials were associated with larger overall display changes. As Miller (1989) pointed out, the total amount of display change is an important factor in the control of visual attention (see also MartinEmerson \& Kramer, 1997). If these changes modulated capture by onset distractors, an interaction between onset and experiment should emerge from this analysis.

The main effect of similarity, $F(1,22)=119.64, p<.01$, was comparable in both experiments (no Similarity $\times$ Experiment interaction). There did seem to be some interference specifically associated with onset distractors, but even pooled over two experiments and 24 participants, it only approached significance, $F(1$, $22)=3.88, p<.10$. As is clear from Tables 1 and 2 , the effect of onset was small, particularly when compared with the magnitude of the similarity effect. The effect of onset did not interact with experiment, which indicated that introducing the offsets was a valid manipulation to avoid the confound of a predictable additional placeholder.

\section{Discussion}

Experiment 2 replicated and extended the effects reported in Experiment 1. Manual responses were mainly affected by the target similarity of the additional distractor; the effect of abrupt onset was small and not statistically reliable. Attentional capture by abrupt onsets was small when compared with the interference caused by similar distractors. This finding is largely in accord with the contingent capture hypothesis (Folk et al., 1992, 1994). However, when an eye movement to the target was required, there was strong oculomotor capture by abrupt-onset distractors. Again, capture was particularly pronounced when the abrupt-onset distractor was similar to the target. In comparison with the previous experiment, there were more saccades to the similar no-onset distractor: Making the extra placeholder unpredictive of the presence and location of an extra distractor reduced the extent to which (the location of) that placeholder was inhibited.

Capture by abrupt onsets was evident in the accuracy of the saccades but not in the latencies of the manual or saccadic responses. One might speculate that the two motor systems differ in 
their sensitivity to interference by irrelevant onsets. Abrupt onsets may have a privileged status in the oculomotor system and would thus be more salient when making an eye movement than when making a manual response (Findlay \& Walker, 1999). And the more salient a distractor, the more it interferes with responding to the target.

However, manual and saccadic responses differed not only in their motor systems but also in the nature of target localization. In the manual response block, the additional distractor did not actually afford a response. That is, the distractor could not have activated a manual response, whereas it certainly could (and did) activate a saccadic response. In addition, foveating the target required a higher resolution spatial localization than the manual response, which simply required a manual indication of the target quadrant. Also note that inferences on capture for the different types of responses are based on different types of data (latency for manual responses, accuracy for saccades). In fact, there was no evidence of capture by onsets in the correct latencies for either type of response.

Thus, any speculation on the differential sensitivity of manual and saccadic responses to abrupt onsets seems premature. More insight into this issue might be gained with a manual response that can be directed toward the distractor and that requires a finegrained spatial localization of the target. Therefore, in the manual response block of Experiment 3, participants had to move the mouse cursor to the target as quickly and accurately as possible while maintaining fixation.

\section{Experiment 3}

\section{Method}

Participants. Twelve new observers ( 3 men and 9 women; age range: 18-32 years) took part for course credit. All had self-reported normal or corrected-to-normal vision and were able to discriminate the colors used in this experiment.

Materials and stimuli. With regard to the displays, the only change in comparison with Experiment 2 was the continuous illumination of the fixation point. The fixation point remained illuminated to help participants maintain fixation in the manual response block and control the strong tendency to track the mouse cursor. In the manual response block, the position of the (standard crosshair) mouse cursor was sampled at $100 \mathrm{~Hz}$.

Procedure. The task was the same as in the first two experiments: Observers had to indicate the location of the red target among green distractors either with a saccade or manually.

Data analysis. Given the limited temporal resolution with which the mouse cursor was sampled, it was not possible to apply a velocity criterion for a number of consecutive samples to determine the start and the end of a mouse movement (in contrast to saccades). Therefore, we analyzed the (initial) direction of the movement at the point where the mouse cursor exceeded a $1^{\circ}$ ring around fixation. We determined the angle of this point relative to the center of the display and classified the movement as directed to the target, the additional distractor, one of the other distractors, or an empty display segment, in the same way that the saccade endpoints were classified. The latency with which the $1^{\circ}$ imaginary boundary was crossed was taken as the MRT. ${ }^{1}$ These latencies were analyzed in the same way as the saccadic reaction times.

With respect to the saccadic responses, the only change in comparison with the previous experiment was the continuous illumination of the fixation point. The presence or absence of a fixation point is known to influence saccadic latency (Forbes \& Klein, 1996; Reuter-Lorenz, Hughes, \& Fendrich, 1991; Saslow, 1967) and accuracy (e.g., Fischer \& Weber,
1997). We carried out two mixed-factor ANOVAs, with similarity and onset as within-participant factors and experiment as the one betweenparticipant factor: one the mean correct saccadic reaction times and one the transformed probability of directly fixating the target. These analyses were expected to provide insight into how the continuous presence of a foveal fixation point affected the speed and accuracy of oculomotor performance.

\section{Results}

Manual responses. Making the fixation point visible throughout the trial certainly helped participants to maintain fixation (relative to the previous experiments). Only $4.6 \%$ of the trials were rejected because of saccades larger than $2^{\circ}(1.8 \%$ of the baseline trials, $0.7 \%$ of the similar onset trials, $0.8 \%$ of the similar no-onset trials, $0.6 \%$ of the dissimilar onset trials, and $0.8 \%$ of the dissimilar no-onset trials). Of all trials, $8.6 \%$ of the pointing movements were directed to a distractor other than the additional one or to an empty region in the display. Given that these trials were evenly distributed over the different trial types, they were also excluded.

Table 3 lists the accuracy and mean latency of the pointing movements in the different trial types. Participants did not point the cursor toward the additional distractor very often. Note, however, that the pattern is similar to that obtained for the eye movements so far. These error frequencies were not analyzed any further. Clear quantitative evidence for capture was obtained in the latencies of the target-directed responses. Effects of both onset and similarity now emerged, $F(1,11)=7.39, p<.05$, and $F(1,11)=$ 82.58, $p<.01$, respectively: Abrupt onsets and similar distractors interfered with pointing to the target. The effect of onset was much more pronounced in this experiment than in the previous experiments (22- and 15-ms interference with similar and dissimilar onset distractors, respectively). The 1-ms difference between the baseline and dissimilar no-onset trials, $t(11)=1.04, p>.05$, confirmed that, also under these conditions, there was no basic cost of adding an extra element in the display, and target selection was highly efficient.

Saccadic responses. Of all saccadic response trials, $3.6 \%$ were rejected because of improper fixation; $6.1 \%$ as a result of no saccade, too short a saccade, or too large a saccade; and $1.5 \%$ because of anticipatory responses. The majority of saccades were directed toward the target or the additional distractor. Only 1\% landed on one of the other distractors or in an empty region.

Table 3 also list the accuracy and mean latencies of the saccades in the different trial types. The pattern of oculomotor capture was similar to that obtained in the first two experiments: The ANOVA showed significant effects of similarity and onset, $F(1,11)=$ 26.77, $p<.01$, and $F(1,11)=15.96, p<.05$, respectively. In addition, the interaction between both factors was significant, $F(1$,

\footnotetext{
${ }^{1}$ The aim was to have a point of initial direction that was sensitive enough to detect distractor-directed pointing but without picking up random miniature movements of the mouse around fixation. We noticed that extending the criterion to the midpoint between fixation and the ring on which the search items were positioned (which, presumably, was the point at which peak velocity would be reached on average) did not affect the basic pattern of results obtained. Because of corrections during the movement, the number of errors was reduced by using this criterion (but their distribution remained unaltered). In addition, as it took more time to cross the halfway boundary, reaction times were more variable, reducing the power of the latency analysis.
} 
Table 3

Manual and Saccadic Response Data (in Milliseconds) From Experiment 3

\begin{tabular}{lccccr}
\hline & \multicolumn{5}{c}{ Trial type } \\
\cline { 2 - 6 } $\begin{array}{c}\text { Response type } \\
\text { and measure }\end{array}$ & Baseline & $\begin{array}{c}\text { Similar } \\
\text { onset }\end{array}$ & $\begin{array}{c}\text { Similar } \\
\text { no onset }\end{array}$ & $\begin{array}{r}\text { Dissimilar } \\
\text { onset }\end{array}$ & $\begin{array}{r}\text { Dissimilar } \\
\text { no onset }\end{array}$ \\
\hline Manual & & & & & \\
$\quad$ Errors & - & 0.06 & 0.04 & 0.01 & 374 \\
MRT correct & 375 & 454 & 432 & 389 & \\
MRT errors & - & 361 & 467 & 417 & 260 \\
Saccadic & - & 0.30 & 0.09 & 0.01 & \\
$\quad$ Errors & 266 & 312 & 307 & 263 & 198 \\
$\quad$ SRT correct & - & 237 & 263 & \\
SRT errors & & &
\end{tabular}

Note. Errors are the proportions of responses directed to the additional distractor. Dashes indicate no errors could be made in the trials (only applicable to baseline trials). MRT = manual reaction time; $\mathrm{SRT}=$ saccadic reaction time.

$11)=19.29, p<.01$. Similar onsets captured the eyes more frequently than similar no-onset distractors, $T(11)=1.00, p<.01$, and dissimilar onsets, $T(11)=0.00, p<.01$. As in Experiment 2 , the difference between dissimilar onset and no-onset distractors was only marginally significant, $T(3)=0.00, p=.10$. The larger effect of onset when the additional distractor was similar to the target gave rise to the significant interaction between the two factors.

In the latency of the target-directed saccades, only interference from similar distractors was observed, $F(1,11)=83.73, p<.01$. Distractor-directed saccades were initiated faster, and as in both previous experiments, eye movements to irrelevant onsets had the shortest latencies.

The mixed-factor ANOVA on the correct latencies showed a main effect of similarity, $F(1,21)=156.40, p<.01]$ : In both experiments, it took participants longer to initiate the saccade in the presence of a similar distractor. The effect of experiment was also significant, $F(1,21)=5.11, p<.05$, indicating that saccadic latencies were reliably longer with a continuous fixation point.

The same ANOVA on the accuracy data showed the expected effects of similarity and onset, $F(1,22)=101.43, p<.01$, and $F(1,22)=21.08, p<.01$, respectively. The interaction between the two factors also reached significance, $F(1,22)=10.73, p<$ .01. For both similar and dissimilar distractors, onsets were more disruptive than no-onset items, $T(23)=6.50, p<.01$, and $T(6)=$ $0.00, p<.05$, respectively, but the effect of onsets was clearly larger when distractors were similar. More interesting, however, is the main effect of experiment, $F(1,22)=10.73, p<.01$, and its interaction with similarity, $F(1,22)=10.73, p<.01$. The presence of the fixation point helped reduce the amount of oculomotor capture, in particular when the distractor was similar. Two post hoc Mann-Whitney $U$ tests comparing the accuracy between Experiments 2 and 3 in the presence of similar and dissimilar distractors clarified the interaction between experiment and similarity: $(p<.05$ for the similar and $p>.05$ for the dissimilar distractors). However, it is clear that in both experiments, performance with the dissimilar distractors was at ceiling and thus probably not very sensitive to manipulations of central fixation.

\section{Discussion}

Direct capture, spatially responding to the distractor, was found for both responses but was mainly observed in the eye movement condition. However, in spite of the overall difference in error frequencies, the pattern was similar for both manual and saccadic pointing. That is, abrupt-onset distractors disrupted the programming of goal-directed movements, particularly when similar to the target.

If participants managed to suppress overt movements to the distractor, then a slowing of movement latencies to the target resulted. For both responses, the latency increase observed was particularly large when the additional distractor was similar to the target. The interference of abrupt-onset distractors was smaller and was mainly observed in the manual response latencies. With the slower and less ballistic manual movements, it is more likely that the response conflict imposed by the additional distractor is successfully resolved. Yet, resolving this conflict takes time, and distractor interference manifests itself (mainly) in the latencies.

In comparison with Experiment 2 (and Experiment 1, for that matter), abrupt onsets slowed the target-directed manual responses to a much larger extent. This finding suggests that the nature of the response has an effect on the visual characteristics of the distractor that will cause interference. This result is surprising if the computation of a salience map for visual selection is independent of the specific response required. Such independence is (implicitly) assumed in computational models of visual attention, such as Guided Search (Wolfe, 1994) and the theory of visual attention (Bundesen, 1990). For such models to capture the current empirical results, they would have to allow for the possibility that response factors modulate the (attentional) weights of the items in the visual field.

However, the data also seem consistent with the possibility that a dynamic, response-independent representation of salience serves as the basis for manual and saccadic responses. The efficiency of target selection would then be determined by the point in time at which the salience map is probed and the response is made. If one assumes that an onset is very salient initially, but that its potential to compete with the target wears off over time (cf. Theeuwes, Atchley, \& Kramer, 2000), fast responses will be affected more than slower responses. Because saccades are initiated much faster 
than manual button presses, at the time the saccade is made the onset is still salient and captures the eyes on a proportion of trials (but less so when the saccadic latencies are increased by having a foveal fixation point). The button press, however, is initiated at a time when the similar distractor is salient solely by virtue of its target similarity and not because of its abrupt onset. According to this scheme, abrupt onsets interfered with the mouse movements because these were, in general, initiated faster than the manual button presses.

Although the present data do not really allow a proper test of this proposal, the issue can be addressed by comparing the fast and slow manual responses. If timing is the critical issue, onsets should interfere more with the fastest button presses (of Experiment 2) than with the slower ones. This prediction was confirmed in a series of post hoc tests. ${ }^{2}$ However, larger interference with the fast responses was also found in the manual pointing movements, the slowest of which were statistically indistinguishable from the fastest manual button presses (see the Appendix). As a general principle, abrupt onsets seem to interfere with the latency of the fastest responses, and the extent to which the central tendency of the entire distribution is shifted still depends on the magnitude of the onset effect on those fastest responses. This magnitude seems to be modulated by the specific response demands.

The offset of a fixation point prior to or simultaneous with target presentation is known to reduce saccadic latencies (Forbes \& Klein, 1996; Reuter-Lorenz et al., 1991). This fixation offset has been linked to a drop in the activity of fixation cells within the rostral pole of the superior colliculus (SC; Dorris, Pare, \& Munoz, 1997; Munoz \& Wurtz, 1993). The reduced fixation activity leaves the saccade-related burst neurons within the more caudal regions of the SC in a state of advanced preparation, increasing the likelihood of saccade triggering by an incoming stimulus (particularly abrupt onsets). For example, prosaccade errors in an antisaccade task occur most often when the fixation point dissappears 200-250 ms before stimulus onset (Fischer \& Weber, 1997). The analyses of saccadic latencies and accuracy across Experiments 2 and 3 extended these observations to the oculomotor capture paradigm: Without advance oculomotor preparation (when the fixation point remains visible), saccade initiation is delayed, and irrelevant onsets are less likely to capture an eye movement.

\section{General Discussion}

The present experiments demonstrated that stimulus-driven capture by irrelevant onset distractors was modulated by goal-driven control settings: Abrupt onsets that were similar to the target were generally more disruptive than dissimilar ones. This effect was observed directly in the eye movements: Observers fixated similar onset distractors much more frequently than dissimilar onsets. Manual aiming movements with the mouse gave a qualitatively similar pattern of results. That is, distractor-directed pointing was most likely to occur on similar onset trials. However, direct capture of these responses was not very frequent. The extent to which irrelevant onsets interfered indirectly with the target-directed manual responses (increasing their latency) depended on the type of manual response required. No reliable interference from abrupt onsets was observed when the target quadrant had to be indicated with a button press, but interference emerged when participants had to direct the mouse cursor to the target.

\section{Control Over Capture}

Attentional cuing studies have consistently shown the dominance of top-down control over stimulus-driven capture by abrupt onsets (Folk \& Remington, 1998; Folk et al., 1992, 1994; Remington et al., 2001). As we outlined in the introduction, observers are generally less capable of ignoring irrelevant onsets in search paradigms (Theeuwes, 1994; Yantis \& Jonides, 1984, 1990). Our results suggest that the discrepancies between the findings of these two lines of research may be due to methodological differences, some of which are outlined below.

For a comparison with our study, the onset cue, colour target conditions of Experiments 1 and 3 of Folk et al. (1992) are most relevant. Observers searched for a color target (a red $x$ or $=$ among white $x \mathrm{~s}$ or $=\mathrm{s}$ ). Prior to the search display, an abrupt-onset cue was briefly presented. The validity of this cue was systematically manipulated in Experiment 1; it was completely unpredictive of the target location in Experiment 3. In neither experiment was a validity effect obtained, which was taken as evidence that the cue did not capture attention: Apparently, observers were able to ignore the irrelevant onset. However, it is important to note that the abrupt onset preceded the search display by $100 \mathrm{~ms}$, a delay after which considerable top-down control may be possible (Kim \& Cave, 1999; Theeuwes et al., 1999, 2000). Also, the combination of a specific type of cue with a specific type of target was blocked in the cuing studies, which might have made it easier for participants to ignore the irrelevant cue when it did not share target features. In addition, the cue could not be responded to, something that we have shown to be an important factor. It seems that under these experimental conditions top-down control is simply more potent in overriding stimulus-driven factors than in the search studies, but that is not to say that capture is never purely stimulusdriven (Yantis, 1993).

Folk and Remington (1998) explained the discrepancy between their results and the search studies in terms of nonspatial interference. In their study, an irrelevant color singleton cue did not result in a validity effect, indicating that spatial attention was not captured, but did cause a general slowing down. The authors argued that a similar effect could be responsible for the interference caused by irrelevant onset distractors in visual search. Studies

\footnotetext{
${ }^{2}$ For each individual participant in Experiments 2 and 3, a median split was performed on the correct MRTs. The 50\% fastest and 50\% slowest responses were used to calculate a fast and a slow mean for every trial type (see the Appendix). Four ANOVAs were performed with similarity and onset-no onset as within-participant factors: one for each of the fast and slow responses of both experiments. For the fast responses of Experiment 2 , there were main effects of onset and similarity, $F(1,11)=6.20, p<.05$, and $F(1,11)=36.67, p<.01$, respectively. Only the effect of similarity remained when the slow responses were analyzed, $F(1,11)=33.67, p<$ .01. The same pattern was observed for the fast and slow responses of Experiment 3: There were main effects of both onset and similarity on the fast movements, $F(1,11)=18.06, p<.01$, and $F(1,11)=49.73, p<.01$, respectively, but only similar distractors interfered with the slow responses, $F(1,11)=82.65, p<.01$. Note that for both similar and dissimilar onset trials, there were no differences between the slowest responses of Experiment 3 and the fastest responses of Experiment 2, $t(22)=-1.31$ and $t(22)=-0.31$, respectively, $p s>.10$. We thank an anonymous reviewer for suggesting this analysis to study the relation between speed of responding and the magnitude of onset interference.
} 
manipulating the response compatibility of the additional distractor have provided some evidence against this account (Theeuwes \& Burger, 1998; Theeuwes et al., 1999). However, the strong oculomotor capture effects obtained in previous studies and the present experiments are more conclusive: Capture of the eyes (and the prolongation of MRTs that were a result of it in Theeuwes et al., 1999) is obviously spatial.

It may be argued, however, that whereas covert attentional capture is contingent on top-down control, such a dependence does not apply to overt capture of the eyes. Thus, it could be that oculomotor capture is not indicative of attentional capture. The extent to which covert attention and eye movements are linked is an issue that has been hotly debated over the last two decades. The most extreme position in this regard is the premotor theory of attention, which equates attentional shifts with saccade preparation (Rizzolatti, Riggio, Dascola, \& Umilta, 1987; Rizzolatti, Riggio, \& Sheliga, 1994; Sheliga, Riggio, \& Rizzolatti, 1994). The evidence for such an obligatory link between attention and saccade preparation is inconsistent (e.g., Klein, 1980; Klein \& Pontefract, 1994). However, several studies, using different paradigms, have shown saccade execution to be preceded by a shift of attention to the location of the target of the eye movement (Deubel \& Schneider, 1996; Hoffman \& Subramaniam, 1995; Kowler, Anderson, Dosher, \& Blaser, 1995; McPeek, Maljkovic, \& Nakayama, 1999; Shepherd, Findlay, \& Hockney, 1986). On the basis of these findings, we think it is unlikely that oculomotor capture in the present experiments occurred without attentional capture.

The present results may be accounted for in terms of a salience map on which both stimulus-driven and goal-driven factors impinge, as in Wolfe's (1994) Guided Search, for example. Such an account can also naturally accommodate findings from previous search and attentional cuing studies. However, our results also suggest that the computation of such a spatial representation of salience is modulated by the specific action directed to the target.

\section{Response-Dependent Interference}

In addition to showing the contribution of both stimulus-driven and goal-driven factors within a single paradigm, the present experiments also demonstrated the importance of response factors in studying visual selective processing. The interference from abrupt-onset distractors was only marginal when observers had to manually indicate in which quadrant the target fell (Experiments 1 and 2). However, when (in Experiment 3 ) the distractor afforded a manual response and the target had to be localized with much more precision, interference from abrupt onsets was much more pronounced (mainly in latency but also in accuracy to a limited extent)

Meegan and Tipper (1999) demonstrated interference from distractors in a selective reaching task. In their study, when participants reached to a target that differed from a background of homogeneous distractors in a single feature, strong distractor interference was observed. Distractors close and ipsilateral to the hand used for reaching were particularly disruptive. When an obstacle was placed in front of the disruptive distractor, which would hinder responding to it, interference was reduced. Of particular relevance here, when the response was to verbally indicate the target location, interference was eliminated. The authors argued that these manipulations affected the extent to which distrac- tors induced visuomotor competition: The more a distractor affords responding to, the more it competes for action and the stronger it interferes with target-directed action (see also Keele, 1972).

One possible explanation for the current results is that abrupt onsets directly induced visuomotor competition, which was modulated by whether the onset could be responded to. The work in selective reaching and the present experiments show that what seems like a similar task on an abstract level can yield different patterns of results (and therefore can lead to different conclusions), depending on the response required.

The different results obtained with the two manual responses studied in these experiments were not due to mouse pointing simply being more sensitive in detecting interference from additional distractors than the button press response because only the effect of abrupt onset was modulated by the particular manual response. A possible explanation is that manipulating the target similarity and the onset of an additional distractor differentially affected vision for identification and vision for action systems, which jointly determine the eventual response. That is, the similarity effect may be related to the more pure perceptual process of discriminating the target from distractors. Adding a similar (onset or no-onset) distractor to the display decreased the targetdistractor discriminability, independent of the type of response required. Abrupt onsets are likely to activate the magnocellular portion of the visual system that preferentially projects to parietal visuomotor areas that control eye and hand movements (Colby \& Goldberg, 1999; Milner \& Goodale, 1995). The responsivity of these areas may be modulated by the specifics of the required output.

\section{Saccade Target Selection}

Theeuwes et al. (1999) explained stimulus-driven capture of the eyes in terms of a race between the programming of a reflexive, stimulus-driven saccade to the onset and a voluntary, goal-driven saccade to the target. This explanation ties in with the idea of two distinct, parallel neural pathways for the generation of different types of saccades (Gaymard, Ploner, Rivaud, Vermersch, \& Pierrot-Deseilligny, 1998). Stimulus-driven, visually guided saccades are thought to be mediated by a parieto-tectal pathway (from parietal eye fields to the SC), whereas goal-driven saccades would depend on the frontal eye fields (FEF) and their projections to brainstem saccade-generating structures (directly and indirectly via the $\mathrm{SC}$ ).

The modulation of stimulus-driven oculomotor capture by color similarity appears to be at odds with such a scheme. If eye movements to the onset distractors are stimulus-driven saccades mediated by the parieto-tectal pathway, this system must somehow be set to respond to abrupt onsets in the target color. Such a process does not seem very likely, given that the major input into this pathway is magnocellular and thus sensitive to luminance, but not wavelength, information (Schiller, 1998).

However, it may be that the entire population of saccades to the similar onset distractor actually consists of two subpopulations: one group of genuine stimulus-driven saccades mediated by the parieto-tectal system and another group solely driven by top-down salience mediated by the FEF. Alternatively, it may be that bottom-up salience is combined with top-down information some- 
where in the system. Recent neurophysiological studies on the interaction between the FEF and SC (Hanes \& Wurtz, 2001; Sommer \& Wurtz, 2000) have indicated that both structures are involved in programming stimulus-driven and goal-driven saccades. For instance, saccade-related FEF neurons that project directly to the SC increase their discharge for erroneous prosaccades in an antisaccade task (Everling \& Munoz, 2000). Such prosaccade errors have often been regarded as reflexive, stimulus-driven saccades mediated by the SC that need to be inhibited (by frontal control systems) for successful antisaccade performance (Guitton, Butchel, \& Douglas, 1985). In addition, the SC is involved in programming the correct antisaccades (Everling, Dorris, Klein, \& Munoz, 1999). It seems that although on a behavioral level one can distinguish between different types of saccades (stimulus-driven vs. goal-driven), the structures and pathways involved in their programming and execution do not seem to be as segregated as is often assumed. Therefore, given the interactive nature of the saccadic system, saccade programming may well be determined by the competitive interactions within a single oculomotor salience map (which does not have to be confined to a single neural locus!). We cannot distinguish the two accounts behaviorally on the basis of the present findings.

In everyday life, humans are confronted with a wide range of possible objects on which to act (fixate, point, reach, grasp, etc.). Successful interaction with the environment requires selection of the relevant objects for action. Orienting toward objects that suddenly appear in the visual scene is probably an adaptive response. However, to prevent continuous distraction by irrelevant items that happen to be salient, some top-down control is necessary. The current study demonstrates the influence of both of these processes, but the extent of their impact depends on the nature of the response.

\section{References}

Anscombe, F. J. (1948). The transformation of Poisson, binomial, and negative binomial data. Biometrika, 35, 246-254.

Bacon, W. F., \& Egeth, H. E. (1994). Overriding stimulus-driven attentional capture. Perception \& Psychophysics, 55, 485-496.

Briand, K. A., Larrison, A. L., \& Sereno, A. B. (2000). Inhibition of return in manual and saccadic response systems. Perception \& Psychophysics, 62, 1512-1524.

Bundesen, C. (1990). A theory of visual attention. Psychological Review, 97, 523-547.

Colby, C. L., \& Goldberg, M. E. (1999). Space and attention in parietal cortex. Annual Review of Neuroscience, 22, 319-349.

Deubel, H., \& Schneider, W. X. (1996). Saccade target selection and object recognition: Evidence for a common attentional mechanism. Vision Research, 36, 1827-1837.

Dorris, M. C., Pare, M., \& Munoz, D. P. (1997). Neuronal activity in monkey superior colliculus related to the initiation of saccadic eye movements. Journal of Neuroscience, 17, 8566-8579.

Egeth, H. E., \& Yantis, S. (1997). Visual attention: Control, representation, and time course. Annual Review of Psychology, 48, 269-297.

Everling, S., Dorris, M. C., Klein, R. M., \& Munoz, D. P. (1999). Role of primate superior colliculus in preparation and execution of anti-saccades and pro-saccades. Journal of Neuroscience, 19, 2740-2754.

Everling, S., \& Munoz, D. P. (2000). Neuronal correlates for preparatory set associated with pro-saccades and anti-saccades in the primate frontal eye field. Journal of Neuroscience, 20, 387-400.

Findlay, J. M., \& Walker, R. (1999). A model of saccade generation based on parallel processing and competitive inhibition. Behavioral \& Brain Sciences, 22, 661-721.

Fischer, B., \& Weber, H. (1997). Effects of stimulus conditions on the performance of antisaccades in man. Experimental Brain Research, 116, 191-200.

Folk, C. L., \& Remington, R. (1998). Selectivity in distraction by irrelevant featural singletons: Evidence for two forms of attentional capture. Journal of Experimental Psychology: Human Perception and Performance, 24, 847-858.

Folk, C. L., Remington, R. W., \& Johnston, J. C. (1992). Involuntary covert orienting is contingent on attentional control settings. Journal of Experimental Psychology: Human Perception and Performance, 18, $1030-1044$.

Folk, C. L., Remington, R. W., \& Wright, J. H. (1994). The structure of attentional control: Contingent attentional capture by apparent motion, abrupt onset, and color. Journal of Experimental Psychology: Human Perception and Performance, 20, 317-329.

Forbes, K., \& Klein, R. M. (1996). The magnitude of the fixation offset effect with endogenously and exogenously controlled saccades. Journal of Cognitive Neuroscience, 8, 344-352.

Gaymard, B., Ploner, C. J., Rivaud, S., Vermersch, A. I., \& PierrotDeseilligny, C. (1998). Cortical control of saccades. Experimental Brain Research, 123, 159-163.

Gellatly, A., \& Cole, G. (2000). Accuracy of target detection in new-object and old-object displays. Journal of Experimental Psychology: Human Perception and Performance, 26, 889-899.

Gellatly, A., Cole, G., \& Blurton, A. (1999). Do equiluminant object onsets capture visual attention? Journal of Experimental Psychology: Human Perception and Performance, 25, 1609-1624.

Gibson, B. S. (1996a). Visual quality and attentional capture: A challenge to the special role of abrupt onsets. Journal of Experimental Psychology: Human Perception and Performance, 22, 1496-1504.

Gibson, B. S. (1996b). The masking account of attentional capture: A reply to Yantis and Jonides (1996). Journal of Experimental Psychology: Human Perception and Performance, 22, 1514-1520.

Guitton, D., Butchel, H. A., \& Douglas, R. M. (1985). Frontal lobe lesions in man cause difficulties in suppressing reflexive glances and in generating goal-directed saccades. Experimental Brain Research, 58, 455472.

Hanes, D. P., \& Wurtz, R. H. (2001). Interaction of the frontal eye field and superior colliculus for saccade generation. Journal of Neurophysiology, $85,804-815$.

Hoffman, J. E., \& Subramaniam, B. (1995). The role of visual-attention in saccadic eye-movements. Perception \& Psychophysics, 57, 787-795.

Irwin, D. E., Colcombe, A. M., Kramer, A. F., \& Hahn, S. (2000). Attentional and oculomotor capture by onset, luminance and color singletons. Vision Research, 40, 1443-1458.

Keele, S. W. (1972). Attention demands of memory retrieval. Journal of Experimental Psychology, 93, 245-248.

Kim, M.-S., \& Cave, K. R. (1999). Top-down and bottom-up attentional control: On the nature of interference from a salient distractor. Perception \& Psychophysics, 61, 1009-1023.

Klein, R. M. (1980). Does oculomotor readiness mediate cognitive control of visual attention? In R. S. Nickerson (Ed.), Attention and performance VIII (pp. 259-276). Hillsdale, NJ: Erlbaum.

Klein, R. M., \& Pontefract, A. (1994). Does oculomotor readiness mediate cognitive control of visual attention? Revisited! In C. Umilta \& M. Moscovitch (Eds.), Attention and performance XV (pp. 333-350). Cambridge, MA: MIT Press.

Kowler, E., Anderson, E., Dosher, B., \& Blaser, E. (1995). The role of attention in the programming of saccades. Vision Research, 35, 18971916.

Martin-Emerson, R., \& Kramer, A. F. (1997). Offset transients modulate 
attentional capture by sudden onsets. Perception \& Psychophysics, 59, 739-751.

McPeek, R. M., Maljkovic, V., \& Nakayama, K. (1999). Saccades require focal attention and are facilitated by a short-term memory system. Vision Research, 39, 1555-1566.

Meegan, D. V., \& Tipper, S. P. (1999). Visual search and target-directed action. Journal of Experimental Psychology: Human Perception and Performance, 25, 1347-1362.

Miller, J. (1989). The control of attention by abrupt visual onsets and offsets. Perception \& Psychophysics, 45, 567-571.

Milner, A. D., \& Goodale, M. A. (1995). The visual brain in action. Oxford, England: Oxford University Press.

Munoz, D. P., \& Wurtz, R. H. (1993). Fixation cells in monkey superior colliculus: 1. Characteristics of cell discharge. Journal of Neurophysiology, 70, 559-575.

Remington, R. W., Folk, C. L., \& McLean, J. P. (2001). Contingent attentional capture or delayed allocation of attention? Perception \& Psychophysics, 63, 298-307.

Reuter-Lorenz, P. A., Hughes, H. C., \& Fendrich, R. (1991). The reduction of saccadic latency by prior offset of the fixation point: An analysis of the gap effect. Perception \& Psychophysics, 49, 167-175.

Rizzolatti, G., Riggio, L., Dascola, I., \& Umilta, C. (1987). Reorienting attention across the horizontal and vertical meridians: Evidence in favor of a premotor theory of attention. Neuropsychologia, 25, 31-40.

Rizzolatti, G., Riggio, L., \& Sheliga, B. M. (1994). Space and selective attention. In C. Umilta \& M. Moscovitch (Eds.), Attention and performance $X V$ (pp. 231-265). Cambridge, MA: MIT Press.

Saslow, M. G. (1967). Effects of components of displacement-step stimuli upon latency for saccadic eye movement. Journal of the Optical Society of America, 57, 1024-1029.

Schiller, P. H. (1998). The neural control of visually guided eye movements. In J. E. Richards (Ed.), Cognitive neuroscience of attention: A developmental perspective (pp. 3-50). London: Erlbaum.

Sheliga, B. M., Riggio, L., \& Rizzolatti, G. (1994). Orienting of attention and eye movements. Experimental Brain Research, 98, 507-522.

Shepherd, M., Findlay, J. M., \& Hockney, R. J. (1986). The relationship between eye movements and spatial attention. Quarterly Journal of Experimental Psychology: Human Experimental Psychology, 38(A), 475-491.

Simons, D. J. (2000). Attentional capture and inattentional blindness. Trends in Cognitive Sciences, 4, 147-155.
Sommer, M. A., \& Wurtz, R. H. (2000). Composition and topographic organization of signals sent from the frontal eye field to the superior colliculus. Journal of Neurophysiology, 83, 1979-2001.

Theeuwes, J. (1994). Stimulus-driven capture and attentional set: Selective search for color and visual abrupt onsets. Journal of Experimental Psychology: Human Perception and Performance, 20, 799-806.

Theeuwes, J., Atchley, P., \& Kramer, A. F. (2000). On the time-course of top-down and bottom-up control of visual attention. In S. Monsell \& J. Driver (Eds.), Attention and performance XVIII (pp. 105-124). Cambridge, MA: MIT Press.

Theeuwes, J., \& Burger, R. (1998). Attentional control during visual search: The effect of irrelevant singletons. Journal of Experimental Psychology: Human Perception and Performance, 24, 1342-1353.

Theeuwes, J., Kramer, A. F., Hahn, S., \& Irwin, D. E. (1998). Our eyes do not always go where we want them to go: Capture of the eyes by new objects. Psychological Science, 9, 379-385.

Theeuwes, J., Kramer, A. F., Hahn, S., Irwin, D. E., \& Zelinsky, G. J. (1999). Influence of attentional capture on oculomotor control. Journal of Experimental Psychology: Human Perception and Performance, 25, 1595-1608.

Treisman, A. M., \& Gelade, G. (1980). A feature-integration theory of attention. Cognitive Psychology, 12, 97-136.

Watson, D. G., \& Humphreys, G. W. (1997). Visual marking: Prioritizing selection for new objects by top-down attentional inhibition of old objects. Psychological Review, 104, 90-122.

Wolfe, J. M. (1994). Guided search 2.0: A revised model of visual search. Psychonomic Bulletin \& Review, 1, 202-238.

Yantis, S. (1993). Stimulus-driven attentional capture and attentional control settings. Journal of Experimental Psychology: Human Perception and Performance, 19, 676-681.

Yantis, S., \& Hillstrom, A. P. (1994). Stimulus-driven attentional capture: Evidence from equiluminant visual objects. Journal of Experimental Psychology: Human Perception and Performance, 20, 95-107.

Yantis, S., \& Jonides, J. (1984). Abrupt visual onsets and selective attention: Evidence from visual search. Journal of Experimental Psychology: Human Perception and Performance, 10, 601-620.

Yantis, S., \& Jonides, J. (1990). Abrupt visual onsets and selective attention: Voluntary versus automatic allocation. Journal of Experimental Psychology: Human Perception and Performance, 16, 121-134.

\section{Appendix}

Manual Response Latencies (in Milliseconds) in Experiments 2 and 3 After a Median Split to Calculate Means for the Fast and the Slow Half of the Responses

\begin{tabular}{ccccc}
\hline & \multicolumn{4}{c}{ Trial type } \\
\cline { 2 - 5 } Manual response & $\begin{array}{c}\text { Similar } \\
\text { onset }\end{array}$ & $\begin{array}{c}\text { Similar } \\
\text { no onset }\end{array}$ & $\begin{array}{c}\text { Dissimilar } \\
\text { onset }\end{array}$ & $\begin{array}{c}\text { Dissimilar } \\
\text { no onset }\end{array}$ \\
\hline Experiment 2 & & & & \\
50\% fastest & 457 & 444 & 409 & 395 \\
50\% slowest & 600 & 607 & 538 & 537 \\
Experiment 3 & & & & \\
50\% fastest & 402 & 367 & 354 & 335 \\
$50 \%$ slowest & 486 & 480 & 414 & 405 \\
\hline
\end{tabular}

